\title{
Editorial
}

\section{Education and Research in Medical Institutions in India}

This issue of the Annals opens with two important and most relevant articles related to medical education and research in India. Prof. Shridhar Sharma is a veteran medical educationist and administrator. He and his colleague has traced the history and described the current status of medical education in India. This is an excellent treatise on the existing health scenario in the country, including the National health status indicators and highlighting the current health system in the country. Author has delved into the history of medical education in India starting from the philosophy of Ayurveda prevalent from 800 BC onwards. Siddha and Unani medicine have also been touched. The main review, however focuses on growth of medical education in Independent India with respect to allopathic system. ROME (reorientation of medical education), now into history, was a significant venture taken in collaboration with World Health Organization (WHO) in 1977. Each medical college was supposed to establish a well-knit rural referral system. However, lack of necessary infrastructure, logistics, and poor motivation of faculty led to only a partial success. The main emphasis on community-oriented training was also not fulfilled. National Health Policy (1983) was the next step to streamline medical education in India. Efforts following the release of this policy are discussed in detail. The article then goes on to discuss the Medical Council of India, its regulations for selection of medical students, teachers and their promotions, existing curriculum and objectives of graduate medical education. Dr. Sharma has very aptly analyzed the strengths and weakness of the present system of medical education in India. Inadequacy of faculty development is also highlighted. The paper ends with the issues and predictions for future. Professor Sharma concluded with the need for a better equipped physician in the 21 st century.

The second article by Ghosh and Ghosh touches on the other side of the coin: research in medical colleges. Authors believe that several factors are responsible for poor quality of research in these institutions, based on evidence generated from various studies and papers published in indexed journals between 1985 to 2017. These factors were identified as poor infrastructure, high patient load, lack of faculty members with adequate research exposure, private practice, lack of incentive, motivation and funds for research. Authors feel that the quality of teachers in medical colleges needs much to be desired. They are particularly not tuned and trained for carrying and supervising research. The last part of the article focuses on the solutions suggested by the authors; and how can the faculty in medical colleges be engaged in research. A heavy emphasis has been laid on to augmenting and changing the way community medicine is being taught.

In a nutshell, both these papers by Dr. Sharma and by Ghosh and Ghosh very aptly describe the current scenario and bottlenecks in medical education and research in Indian medical schools. Authors of both papers have called for a major reforms in the medical curriculum, so as to benefit the physician and the community he/she is going to serve as a benefactor. 
Besides these two main articles, there are papers from other specialties including neurology, otorhinolaryngology, and orthopedics.

In the first specialty article for this issue, UK Misra from SGPGIMS, Lucknow reviews the clinical and experimental studies in Japanese Encephalitis. The author summarizes the important clinical, radiological, neurophysiologic and biochemical studies. A syndromic approach is advised to categorize the patient of acute encephalitic syndrome (AES) into neurologic and systemic group. Acyclovir therapy is recommended for the neurologic group. In systematic group, treatment with doxycycline for scrub typhus, artesunate for malaria, ceftriaxone for leptospira, and fluid management for dengue are recommended.

Saurabh Varshney discusses their experience of treating 72 cases of pituitary tumor by endoscopic trans-nasal trans-sphenoidal approach between 2015-2016 in adults. Complications rate was less than $10 \%$. Author concluded that endoscopic trans-sphenoidal approach is less traumatic and permits good visualization with overall reduced hospital stay and lesser complications.

The next article by Nair et al from the Department of Orthopedics, AIIMS, Delhi bring about the first of its kind systemic study describing the clinical aspects, prevalence, and imaging features of adhesive capsulitis of the shoulder (ACS) in 16 patients with type-1 diabetes. Presence of retinopathy and limited joint mobility were recognized as independent predictors for ACS in these patients. Imaging confirms the diagnosis and facilitates institution of early therapy.

Sharma et al from AIIMS, Jodhpur report a case of 23-year-old primigravida at 32 weeks gestation with complex congenital heart disease. Her course during the pregnancy is described. Despite a turbulent hospital stay, she delivered a live boy without any complications. The newborn was healthy and did not have a heart defect. Favorable outcome could be achieved by a meticulous planning and multidisciplinary team management.

To end this issue, we present a mini-review by Jyotsna Kailashiya from the biochemistry department of Institute of Medical Sciences, Banaras Hindu University on platelet-derived micorparticles (PMPs). These particles have been proposed as potential biomarkers for several conditions such as myocardial infarction, stroke, venous thrombo-embolism, etc. Biosensors are new analytic tools that are now being designed for analysis of PMPs. Author compiles these designs and discusses their pros and cons.

Hope you will enjoy reading this issue.

Dr. Kuldeep Singh 